\title{
Primality Proving via One Round in ECPP and One Iteration in AKS
}

\author{
Qi Cheng* \\ School of Computer Science \\ The University of Oklahoma \\ Norman, OK 73019, USA \\ qcheng@cs.ou.edu
}

\begin{abstract}
On August 2002, Agrawal, Kayal and Saxena announced the first deterministic and polynomial time primality testing algorithm. For an input $n$, the AKS algorithm runs in heuristic time $\tilde{O}\left(\log ^{6} n\right)$. Verification takes roughly the same amount of time. On the other hand, the Elliptic Curve Primality Proving algorithm (ECPP), runs in random heuristic time $\tilde{O}\left(\log ^{6} n\right)\left(\tilde{O}\left(\log ^{5} n\right)\right.$ if the fast multiplication is used), and generates certificates which can be easily verified. More recently, Berrizbeitia gave a variant of the AKS algorithm, in which some primes cost much less time to prove than a general prime does. Building on these celebrated results, this paper explores the possibility of designing a more efficient algorithm. A random primality proving algorithm with heuristic time complexity $\tilde{O}\left(\log ^{4} n\right)$ is presented. It generates a certificate of primality which is $O(\log n)$ bits long and can be verified in deterministic time $\tilde{O}\left(\log ^{4} n\right)$. The reduction in time complexity is achieved by first generalizing Berrizbeitia's algorithm to one which has higher density of easily-proved primes. For a general prime, one round of ECPP is deployed to reduce its primality proof to the proof of a random easilyproved prime.
\end{abstract}

\section{Introduction}

Testing whether a number is prime or not is one of the fundamental problems in computational number theory. It has wide applications in computer science, especially in cryptography. After tremendous efforts invested by researchers in about two hundred years, it was finally proved by Agrawal, Kayal and Saxena [3] that the set of primes is in the complexity class $\mathbf{P}$. For a given integer $n$, the AKS algorithm runs in time no longer than $\tilde{O}\left(\log ^{12} n\right)$, while the best deterministic algorithm before it has subexponential complexity [2]. Under a conjecture concerning the density of the Sophie-Germain primes, The AKS algorithm should give out answer in time $\tilde{O}\left(\log ^{6} n\right)$.

Notation: In this paper, we use "ln" for logarithm base $e$ and "log" for logarithm base 2. We write $r^{\alpha}|| n$, if $r^{\alpha} \mid n$ but $r^{\alpha+1} \nmid n$. By $\tilde{O}(f(n))$, we mean $O(f(n)$ polylog $(f(n)))$.

^ This research is partially supported by NSF Career Award CCR-0237845

D. Boneh (Ed.): CRYPTO 2003, LNCS 2729, pp. 338 348 2003.

(C) International Association for Cryptologic Research 2003 
The AKS algorithm is based on the derandomization of a polynomial identity testing. It involves many iterations of polynomial modular exponentiation. To test the primality of a integer $n$, the algorithm first searches for a suitable $r$, which is provably $O\left(\log ^{6} n\right)$, or heuristically $O\left(\log ^{2} n\right)$. Then the algorithm will check for $l$ from 1 to $L=\lceil 2 \sqrt{r} \log n\rceil$, whether

$$
(x+l)^{n}=x^{n}+l \quad\left(\bmod n, x^{r}-1\right) .
$$

The algorithm declares that $n$ is a prime if all the checks pass. The computing of $(x+l)^{n}\left(\bmod n, x^{r}-1\right)$ takes time $\tilde{O}\left(r \log ^{2} n\right)$ if we use the fast multiplication. The total time complexity is thus $\tilde{O}\left(r L \log ^{2} n\right)$.

While the AKS algorithm is a great accomplishment in the theory, the current version is very slow. Unless its time complexity can be dramatically improved, it cannot replace random primality testing algorithms with better efficiency. In most of applications in cryptography, an efficient random algorithm is sufficient, as long as the algorithm can generate a certificate of primality, which in deterministic time convinces a verifier who does not believe any number theory conjectures. A primality testing algorithm which generates a certificate of primality is sometimes called primality proving algorithm. Similarly a primality testing algorithm which generates a certificate of compositeness is sometimes called compositeness proving algorithm. Very efficient random compositeness proving algorithms have long been known. Curiously, primality proving algorithms 5, 1. lag far behind of compositeness proving algorithms in term of efficiency and simplicity.

Recently, Berrizbeitia 7] proposed a brilliant modification to the AKS original algorithm. He used the polynomial $x^{2^{s}}-a$ instead of $x^{r}-1$ in equation (1), where $2^{s} \approx \log ^{2} n$. Among others, he was able to prove the following proposition:

Proposition 1. Given an integer $n \equiv 1(\bmod 4)$. Denote $s=\lceil 2 \log \log n\rceil$. Assume that $2^{k}|| n-1$ and $k \geq s$. If there exists an integer $a$, such that $\left(\frac{a}{n}\right)=-1$ and $a^{\frac{n-1}{2}} \equiv-1(\bmod n)$, then

$$
(1+x)^{n} \equiv 1+x^{n} \quad\left(\bmod n, x^{2^{s}}-a\right)
$$

iff $n$ is a power of a prime.

Unlike the AKS algorithm, where each prime costs roughly the same, there are "easily-proved primes" in Berrizbeitia's algorithm, namely, the primes $p$ where $p-1$ has a factor of a power of two larger than $\log ^{2} n$. For those primes, one iteration of polynomial modular exponentiation, which runs in time $\tilde{O}\left(\log ^{4} n\right)$, establishes the primality of $p$, provided that a suitable $a$ exists. In fact, $a$ can be found easily if $n$ is indeed a prime and randomness is allowed in the algorithm. It serves as a prime certificate for $n$.

Definition 1. In this paper, for a primality proving algorithm, we call a prime $p$ easily-proved, if the algorithm runs in expected time $\tilde{O}\left(\log ^{4} p\right)$ on $p$. 
What is the density of the easily-proved primes in Berrizbeitia's algorithm? The number of primes of form $2^{s} x+1$ less than $b$ is about $\frac{\pi(b)}{\phi\left(2^{s}\right)}$. Hence heuristically for a random prime $p, p-1$ has a factor $2^{s} \approx \log ^{2} p$ with probability $\frac{1}{2 \log ^{2} p}$, in the other words, the easily-proved primes have density $\frac{1}{2 \log ^{2} p}$ around $p$ in his algorithm.

\subsection{Increasing the Density of Easily-Proved Primes}

We prove the following theorem in Section 5, which can be regarded as a generalization of Proposition 11

Theorem 1. (Main) Given a number $n$ which is not a power of an integer. Suppose that there exists a prime $r, r^{\alpha}|| n-1(\alpha \geq 1)$ and $r \geq \log ^{2} n$. In addition, there exists a number $1<a<n$, such that $a^{r^{\alpha}} \equiv 1(\bmod n), \operatorname{gcd}\left(a^{r^{\alpha-1}}-1, n\right)=$ 1 , and

$$
(1+x)^{n}=1+x^{n} \quad\left(\bmod n, x^{r}-a\right)
$$

then $n$ is a prime.

The number $a$ can be found easily if $n$ is a prime and randomness is allowed. It serves as a prime certificate for $n$. Base on this theorem, we propose a random algorithm which establishes the primality of $p$ in time $\tilde{O}\left(\log ^{4} p\right)$ if $p-1$ contains a prime factor between $\log ^{2} p$ and $C \log ^{2} p$ for some small constant $C$.

Definition 2. We call a positive integer $n C$-good, if $n-1$ has a prime factor $p$ such that $\log ^{2} n \leq p \leq C \log ^{2} n$.

What is the density of $C$-good primes? Clearly the density should be higher than the density of easily-proved primes in Berrizbeitia's algorithm. Let $m=$ $\prod_{p \text { prime } b_{1} \leq p \leq C b_{1}} p$. First we count the number of integers between 1 and $m$ which have a prime factor between $b_{1}$ and $C b_{1}$. This is precisely the number of nontrivial zero-divisors in $\operatorname{ring} \mathbf{Z} / m \mathbf{Z}$ :

$$
(m-1)-m \prod_{p \text { prime }, b_{1} \leq p \leq C b_{1}}\left(1-\frac{1}{p}\right) .
$$

We will prove in Section 4 that this number is greater than $\frac{m}{\ln b_{1}}$ for $C=\mathbf{c}$ and $b_{1}$ sufficiently large, where $\mathbf{c}$ is an absolute constant to be determined later. We need fix a explicit value for $C$ in the algorithm. Without loss of generosity, set $C=2$. For simplicity, we call a number good, when it is 2-good. Since compared with $\log ^{2} n, n$ is very big, we expect that

Conjecture 1. There exists an absolute constant $\lambda$, such that for any sufficiently large integer $n$,

$$
\frac{\text { Number of } 2-\text { good primes between } n-2 \sqrt{n}+1 \text { and } n+2 \sqrt{n}+1}{\text { Number of primes between } n-2 \sqrt{n}+1 \text { and } n+2 \sqrt{n}+1}>\frac{\lambda}{\ln \left(\log ^{2} n\right)} \text {. }
$$

We are unable to prove this conjecture however, but we present in the paper some numerical evidences. We comment that questions about the prime distribution in a short interval are usually very hard. 


\subsection{Algorithm for the General Primes}

For general primes, we apply the idea in the Elliptic Curve Primality Proving algorithm (ECPP). ECPP was proposed by Goldwasser, Kilian [8] and Atkin [4] and implemented by Atkin and Morain [5]. In practice, ECPP performs much better than the current version of AKS. It has been used to prove primality of numbers up to thousands of decimal digits [10].

In ECPP, if we want to prove that an integer $n$ is a prime, we reduce the problem to the proof of primality of a smaller number (less than $n / 2$ ). To achieve this, we try to find an elliptic curve with $\omega n^{\prime}$ points over $\mathbf{Z} / n \mathbf{Z}$, where $\omega$ is completely factored and $n^{\prime}$ is a probable prime greater than $(\sqrt[4]{n}+1)^{2}$. Once we have such a curve and a point on the curve with order $n^{\prime}$, the primality of $n^{\prime}$ implies the primality of $n$. Since point counting on elliptic curves is expensive, we usually use the elliptic curves with complex multiplications of small discriminants. Nonetheless, it is plausible to assume that the order of the curve has the desired form with the same probability as a random integer does. ECPP needs $O(\log n)$ rounds of reductions to eventually reduce the problem to a primality proof of a very small prime, say, less than 1000. As observed in [9, one round of reduction takes heuristic time $\tilde{O}\left(\log ^{5} n\right)$, or $\tilde{O}\left(\log ^{4} n\right)$ if we use the fast multiplication. To get the time complexity, it is assumed that the number of primes between $n-2 \sqrt{n}+1$ and $n+2 \sqrt{n}+1$ is greater than $\sqrt{n} / \log ^{2} n$, and the number of points on an elliptic curve with small discriminant complex multiplication behaves like a random number in the Hassa range. We refer the assumption as the ECPP heuristics. Rigorous proof of the time complexity seems out of reach, as it involves the study of the prime distribution in a short interval.

Our algorithm can be decomposed into two stages. In the first stage, for a general probable prime $n$, we will use one round of ECPP to reduce its proof of primality to a good probable prime $n^{\prime}$ near $n$. For convenience, we require that $n-2 \sqrt{n}+1 \leq n^{\prime} \leq n+2 \sqrt{n}+1$ (See section 6 for implementation issues). Note that up to a constant factor, the time complexity of one round reduction in ECPP is equivalent to the time complexity of finding a curve with a prime order. In the set of primes between $n-2 \sqrt{n}+1$ and $n+2 \sqrt{n}+1$, the density of good primes is $\frac{\lambda}{\ln \left(\log ^{2} n\right)}$ by conjectures. Hence heuristically the extra condition on $n^{\prime}$ (that $n^{\prime}$ should be good) will increase the time complexity merely by a factor of $O(\log \log n)$. Therefore for all the primes, without significant increase of time complexity, we reduce its primality proving to the proof of a good prime. In the second stage, we find a primality certificate for $n^{\prime}$. To do this, we search for $a$ which satisfies the conditions in the main theorem, and compute the polynomial modular exponentiation. The total expected running time of the first and the second stages becomes $\tilde{O}\left(\log ^{4} n\right)$. However, because of the reasons given above, it seems difficult to obtain the rigorous time complexity. Put it altogether, we now have a general purpose prime proving algorithm, which has following properties:

1. it runs very fast $\left(\tilde{O}\left(\log ^{4} n\right)\right)$ assuming reasonable conjectures.

2. For many primes, ECPP subroutine is not needed. 
3. The certificate, which consists of the curve, a point on the curve with order $n^{\prime}, n^{\prime}$ and $a$, is very short. It consists of only $O(\log n)$ bits as opposed to $O\left(\log ^{2} n\right)$ bits in ECPP.

4. A verifier can be convinced in deterministic time $\tilde{O}\left(\log ^{4} n\right)$. In fact, the most time consuming part in the verification is the computation of one polynomial modular exponentiation.

This paper is organized as following: In Section 2, we review the propositions used by AKS and ECPP to prove primality. In Section 3 , we describe our algorithm and present the time complexity analysis. In Section 4 we prove a theorem which can be regarded as an evidence for the density heuristics. The main theorem is proved in Section 5. We conclude this paper with some discussions on the implementation of the algorithm.

\section{Proving Primality in AKS and ECPP}

The ECPP algorithm depends on rounds of reductions of the proof of primality of a prime to the proof of primality of a smaller prime. The most remarkable feature of ECPP is that a verifier who does not believe any conjectures can be convinced in time $\tilde{O}\left(\log ^{3} n\right)$ if the fast multiplication is used. It is based on the following proposition [5].

Proposition 2. Let $N$ be an integer prime to $6, E$ be an elliptic curve over $\mathbf{Z} / N \mathbf{Z}$, together with a point $P$ on $E$ and two integers $m$ and $s$ with $s \mid m$. Denote the infinite point on $E$ by $O$. For each prime divisor $q$ of $s$, denote $(m / q) P$ by $\left(x_{q}: y_{q}: z_{q}\right)$. Assume that $m P=O$ and $\operatorname{gcd}\left(z_{q}, N\right)=1$ for all $q$. If $s>$ $(\sqrt[4]{N}+1)^{2}$, then $N$ is a prime.

The certificate for $N$ in ECPP consists of the curve $E$, the point $P, m, s$ and the certificate of primality of $s$. Usually the ECPP algorithm uses elliptic curves with complex multiplications of small discriminants. For implementation details, see [5].

The AKS algorithm proves a number is a prime through the following proposition.

Proposition 3. Let $n$ be a positive integer. Let $q$ and $r$ be prime numbers. Let $S$ be a finite set of integers. Assume

1. that $q$ divides $r-1$;

2. that $n^{\frac{r-1}{q}} \not \equiv 0,1(\bmod r)$;

3. that $\operatorname{gcd}\left(n, b-b^{\prime}\right)=1$ for all the distinct $b, b^{\prime} \in S$;

4. $\operatorname{that}\left(\begin{array}{c}q+|S|-1 \\ |S|\end{array}\right) \geq n^{2\lfloor\sqrt{r}\rfloor}$;

5. that $(x+b)^{n} \equiv x^{n}+b\left(\bmod x^{r}-1, n\right)$ for all $b \in S$.

Then $n$ is a power of a prime. 


\section{Description and Time Complexity Analysis of Our Algorithm}

Now we are ready to sketch our algorithm.

Input: a positive integer $n$

Output: a certificate of primality of $n$, or "composite".

1. If $n$ is a power of an integer, return "composite".

2. Run a random compositeness proving algorithm, for example, the RabinMiller testing [6, Page 282], on $n$. If a proof of compositeness is found, output the proof, return "composite" and exit;

3. If $n-1$ contains a prime factor between $\log ^{2} n$ and $2 \log ^{2} n$, skip this step. Otherwise, call ECPP to find an elliptic curve over $\mathbf{Z} / n \mathbf{Z}$ with $n^{\prime}$ points, where $n^{\prime}$ is a probable prime and $n^{\prime}$ is 2-good. Set $n=n^{\prime}$;

4. Let $r$ be the prime factor of $n-1$ satisfying $\log ^{2} n \leq r \leq 2 \log ^{2} n$;

5. Randomly select a number $1<b<n$. If $b^{n-1} \neq 1(\bmod n)$, exit;

6. Let $a=b^{\frac{n-1}{r^{\alpha}}}(\bmod n)$; If $a=1$, or $a^{r^{\alpha-1}}=1$, go back to step 5

7. If $\operatorname{gcd}\left(a^{r^{\alpha-1}}-1, n\right) \neq 1$, exit;

8. If $(1+x)^{n} \neq 1+x^{n}\left(\bmod n, x^{r}-a\right)$, exit;

9. Use ECPP procedure to construct the curve and the point and compute the order. Output them with $a$. Return "prime".

On any input integer $n$, this algorithm will either output "composite" with a compositeness proof, or "prime" with a primality proof, or nothing. As we can see, it output nothing, only when the probable prime $n$ in Step 4 is actually composite. The chance should be extremely small.

Now we analyze its time complexity. Testing whether a number $n$ is good or not can be done in time $\tilde{O}\left(\log ^{3} n\right)$. The step 3 takes time $\tilde{O}\left(\log ^{4} n\right)$, if the ECPP heuristics is true, Conjecture 1 in the introduction section is true, and the fast multiplication algorithm is used. If $n$ is indeed a prime, then the probability of going back in step 6 is at most $1 / r$. The step 7 takes time at most $\tilde{O}\left(\log ^{2} n\right)$. The step 8 takes time $\tilde{O}\left(\log ^{4} n\right)$, since $r \leq 2 \log ^{2} n$. Hence the heuristic expected running time of our algorithm is $\tilde{O}\left(\log ^{4} \bar{n}\right)$. Obviously the verification algorithm takes deterministic time $\tilde{O}\left(\log ^{4} n\right)$.

\section{Density of Good Numbers}

What is the probability that a random number has a prime factor between $b_{1}$ and $b_{2}=C b_{1}$ ? Let $m=\prod_{p}$ prime, $b_{1} \leq p \leq b_{2} p$. We first compute the density of integers between 1 and $m-1$ which has a prime factor between $b_{1}$ and $b_{2}$. Those numbers are precisely the zero-divisors in $\mathbf{Z} / m \mathbf{Z}$. The number of nonzero-divisors between 1 and $m$ is $\phi(m)=m \prod_{p \text { prime, } b_{1} \leq p \leq b_{2}}\left(1-\frac{1}{p}\right)$, where $\phi$ is the Euler phi-function. First we estimate the quantity:

$$
\beta_{b_{1}, b_{2}}=\prod_{p \text { prime }, b_{1} \leq i \leq b_{2}}\left(1-\frac{1}{p}\right)
$$


It is known [11] that $\prod_{p<x, p \text { prime }}\left(1-\frac{1}{p}\right)=\frac{e^{-\gamma}}{\ln x}\left(1+O\left(\frac{1}{\ln x}\right)\right)$, where $\gamma$ is the Euler constant. There must exist two absolute constants $c_{1}, c_{2}$, such that

$$
\frac{e^{-\gamma}}{\ln x}\left(1+\frac{c_{1}}{\ln x}\right) \leq \prod_{p<x, p}\left(1-\frac{1}{p}\right) \leq \frac{e^{-\gamma}}{\ln x}\left(1+\frac{c_{2}}{\ln x}\right)
$$

Set $C=\mathbf{c}$ where $\mathbf{c}$ represents $e^{c_{2}-c_{1}+2}$.

$$
\begin{aligned}
\prod_{p \text { prime }, b_{1} \leq p \leq b_{2}}\left(1-\frac{1}{p}\right) & =\frac{\prod_{p \text { prime }, p \leq b_{2}}\left(1-\frac{1}{p}\right)}{\prod_{p \text { prime }, p \leq b_{1}}\left(1-\frac{1}{p}\right)} \\
& \leq \frac{\ln b_{1} \frac{1+\frac{c_{2}}{\ln c_{1}}}{\ln \mathbf{c} b_{1}} \frac{c_{1}}{\ln b_{1}}}{\ln ^{3} b_{1}+\left(2 \ln \mathbf{c}+c_{1}\right) \ln ^{2} b_{1}+\left(\ln ^{2} \mathbf{c}+2 c_{1} \ln \mathbf{c}\right) \ln b_{1}+c_{1} \ln ^{2} \mathbf{c}}
\end{aligned}
$$

Thus $1-\beta_{b_{1}, b_{2}} \geq \frac{\left(\ln \mathbf{c}+c_{1}-c_{2}\right) \ln ^{2} b_{1}-\left(\ln ^{2} \mathbf{c}+2 c_{2} \ln \mathbf{c}\right) \ln b_{1}-c_{2} \ln ^{2} \mathbf{c}}{\ln ^{3} b_{1}+\left(2 \ln \mathbf{c}+c_{1}\right) \ln ^{2} b_{1}+\left(\ln ^{2} \mathbf{c}+2 c_{1} \ln \mathbf{c}\right) \ln b_{1}+c_{1} \ln ^{2} \mathbf{c}}>\frac{1}{\ln b_{1}}$, when $b_{1}$ is sufficiently large. It is expected that the density of good primes in the set of primes in a large interval should not be very far away from $\frac{1}{\ln b_{1}}$. See Table 1 for numerical data concerning the density of 2 -good primes around $2^{500}$. Notice that

$$
\begin{aligned}
\beta_{250000,500000} & =0.9472455 \\
1-\beta_{250000,500000} & =0.0527545 \\
\frac{1}{\ln 250000} & =0.0804556
\end{aligned}
$$

\section{Proof of the Main Theorem}

In this section we prove the main theorem. It is built on a series of lemmas. Some of them are straight-forward generalizations of the lemmas in Berrizbeitia's paper [7. We include slightly different proofs of those lemmas, though, for completeness. Some of the proofs are brief, for details see [7].

Lemma 1. Let $r, p$ be primes, $r \mid p-1$. If $a \in \mathbf{F}_{p}$ is not a $r$-th power of any element in $\mathbf{F}_{p}$, then $x^{r}-a$ is irreducible over $\mathbf{F}_{p}$.

Proof. Let $\theta$ be one of the roots of $x^{r}-a=0$. Certainly $\left[\mathbf{F}_{p}(\theta): \mathbf{F}_{p}\right]>1$. Let $\xi \in \mathbf{F}_{p}$ be one of the $r$-th primitive roots of unity.

$$
x^{r}-a=x^{r}-\theta^{r}=\prod_{0 \leq i \leq r-1}\left(x-\xi^{i} \theta\right) .
$$

Let $\left[\mathbf{F}_{p}(\theta): \mathbf{F}_{p}\right]=r^{\prime}$. Then for all $i,\left[\mathbf{F}_{p}\left(\xi^{i} \theta\right): \mathbf{F}_{p}\right]=r^{\prime}$. Hence over $\mathbf{F}_{p}, x^{r}-a$ will be factored into polynomials of degree $r^{\prime}$ only. Since $r$ is a prime, this is impossible, unless that $r^{\prime}=r$. 
Table 1. Number of 2-good primes around $2^{500}$

\begin{tabular}{|c|c|c|c|c|}
\hline From & To & Number of primes & Number of 2-good primes & Ratio \\
\hline $2^{500}+0$ & $2^{500}+200000$ & 576 & 35 & $6.07 \%$ \\
\hline $2^{500}+200000$ & $2^{500}+400000$ & 558 & 38 & $6.81 \%$ \\
\hline $2^{500}+400000$ & $2^{500}+600000$ & 539 & 30 & $5.56 \%$ \\
\hline $2^{500}+600000$ & $2^{500}+800000$ & 568 & 23 & $4.05 \%$ \\
\hline $2^{500}+800000$ & $2^{500}+1000000$ & 611 & 39 & $6.38 \%$ \\
\hline $2^{500}+1000000$ & $2^{500}+1200000$ & 566 & 26 & $4.59 \%$ \\
\hline $2^{500}+1200000$ & $2^{500}+1400000$ & 566 & 38 & $6.71 \%$ \\
\hline $2^{500}+1400000$ & $2^{500}+1600000$ & 526 & 27 & $5.13 \%$ \\
\hline $2^{500}+1600000$ & $2^{500}+1800000$ & 580 & 26 & $4.48 \%$ \\
\hline $2^{500}+1800000$ & $2^{500}+2000000$ & 563 & 20 & $3.55 \%$ \\
\hline $2^{500}+2000000$ & $2^{500}+2200000$ & 562 & 22 & $3.91 \%$ \\
\hline $2^{500}+2200000$ & $2^{500}+2400000$ & 561 & 21 & $3.74 \%$ \\
\hline $2^{500}+2400000$ & $2^{500}+2600000$ & 609 & 34 & $5.58 \%$ \\
\hline $2^{500}+2600000$ & $2^{500}+2800000$ & 601 & 28 & $4.66 \%$ \\
\hline $2^{500}+2800000$ & $2^{500}+3000000$ & 603 & 33 & $5.47 \%$ \\
\hline $2^{500}+3000000$ & $2^{500}+3200000$ & 579 & 37 & $6.39 \%$ \\
\hline $2^{500}+3200000$ & $2^{500}+3400000$ & 576 & 31 & $5.38 \%$ \\
\hline $2^{500}+3400000$ & $2^{500}+3600000$ & 604 & 35 & $5.79 \%$ \\
\hline $2^{500}+3600000$ & $2^{500}+3800000$ & 612 & 40 & $6.53 \%$ \\
\hline $2^{500}+3800000$ & $2^{500}+4000000$ & 588 & 29 & $4.93 \%$ \\
\hline $2^{500}+4000000$ & $2^{500}+4200000$ & 574 & 33 & $5.75 \%$ \\
\hline $2^{500}+4200000$ & $2^{500}+4400000$ & 609 & 27 & $4.43 \%$ \\
\hline $2^{500}+4400000$ & $2^{500}+4600000$ & 549 & 35 & $6.37 \%$ \\
\hline $2^{500}+4600000$ & $2^{500}+4800000$ & 561 & 30 & $5.34 \%$ \\
\hline $2^{500}+4800000$ & $2^{500}+5000000$ & 545 & 29 & $5.32 \%$ \\
\hline $2^{500}+5000000$ & $2^{500}+5200000$ & 590 & 20 & $3.39 \%$ \\
\hline $2^{500}+5200000$ & $2^{500}+5400000$ & 557 & 27 & $4.84 \%$ \\
\hline $2^{500}+5400000$ & $2^{500}+5600000$ & 591 & 28 & $4.73 \%$ \\
\hline $2^{500}+5600000$ & $2^{500}+5800000$ & 517 & 33 & $6.38 \%$ \\
\hline $2^{500}+5800000$ & $2^{500}+6000000$ & 566 & 18 & $3.18 \%$ \\
\hline $2^{500}+6000000$ & $2^{500}+6200000$ & 575 & 30 & $5.21 \%$ \\
\hline $2^{500}+6200000$ & $2^{500}+6400000$ & 573 & 26 & $4.53 \%$ \\
\hline $2^{500}+6400000$ & $2^{500}+6600000$ & 558 & 36 & $6.45 \%$ \\
\hline $2^{500}+6600000$ & $2^{500}+6800000$ & 574 & 32 & $5.57 \%$ \\
\hline $2^{500}+6800000$ & $2^{500}+7000000$ & 594 & 22 & $3.70 \%$ \\
\hline $2^{500}+7000000$ & $2^{500}+7200000$ & 596 & 31 & $5.20 \%$ \\
\hline $2^{500}+7200000$ & $2^{500}+7400000$ & 567 & 26 & $4.58 \%$ \\
\hline $2^{500}+7400000$ & $2^{500}+7600000$ & 619 & 28 & $4.52 \%$ \\
\hline $2^{500}+7600000$ & $2^{500}+7800000$ & 565 & 25 & $4.42 \%$ \\
\hline $2^{500}+7800000$ & $2^{500}+8000000$ & 561 & 25 & $4.45 \%$ \\
\hline $2^{500}+8000000$ & $2^{500}+8200000$ & 570 & 26 & $4.56 \%$ \\
\hline
\end{tabular}

Lemma 2. Let $n>2$ be an integer. Let $r$ be a prime and $r^{\alpha} \| n-1$. Suppose that there exists a integer $1<a<n$ such that 
1. $a^{r^{\alpha}} \equiv 1(\bmod n)$;

2. $\operatorname{gcd}\left(a^{r^{\alpha-1}}-1, n\right)=1$;

Then there must exist a prime factor $p$ of $n$, such that $r^{\alpha} \| p-1$ and a is not a $r$-th power of any element in $\mathbf{F}_{p}$.

Proof. For any prime factor $q$ of $n, a^{r^{\alpha}} \equiv 1(\bmod q)$ and $a^{r^{\alpha-1}} \not \equiv 1(\bmod q)$, so $r^{\alpha} \mid q-1$. If $r^{\alpha+1} \mid q-1$ for all the prime factors, then $r^{\alpha+1} \mid n-1$, contradiction. Hence there exists a prime factor $p$, such that $r^{\alpha} \| p-1$. Let $g$ be a generator in $\mathbf{F}_{p}^{*}$. If $a=g^{t}$ in $\mathbf{F}_{p}$, then $p-1 \mid t r^{\alpha}$, and $p-1 \not t r^{\alpha-1}$. Hence $r \nmid t$.

In the following text, we assume that $n$ is an integer, $n=p^{l} d$ where $p$ is a prime and $\operatorname{gcd}(p, d)=1$. Assume $r$ is a prime and $r \mid p-1$. Let $x^{r}-a$ be an irreducible polynomial in $\mathbf{F}_{p}$. Let $\theta$ be one of the roots of $x^{r}-a$. For any element in the field $\mathbf{F}_{p}(\theta)$, we can find a unique polynomial $f \in \mathbf{F}_{p}[x]$ of degree less than $r$ such that the element can be represented by $f(\theta)$. Define $\sigma_{m}: \mathbf{F}_{p}(\theta) \rightarrow \mathbf{F}_{p}(\theta)$ as $\sigma(f(\theta))=f\left(\theta^{m}\right)$.

Lemma 3. We have that $a^{m}=a$ in $\mathbf{F}_{p}$ iff $\sigma_{m} \in \operatorname{Gal}\left(\mathbf{F}_{p}(\theta) / \mathbf{F}_{p}\right)$.

Proof. $(\Leftarrow)$ : Since $\sigma_{m} \in \operatorname{Gal}\left(\mathbf{F}_{p}(\theta) / \mathbf{F}_{p}\right), \theta^{m}$ must be a root of $x^{r}-a$. Hence $a=\left(\theta^{m}\right)^{r}=a^{m}$ in $\mathbf{F}_{p}$.

$(\Rightarrow)$ : For any two elements $a, b \in \mathbf{F}_{p}(\theta)$, we need to prove that $\sigma_{m}(a+b)=$ $\sigma_{m}(a)+\sigma_{m}(b)$ and $\sigma_{m}(a b)=\sigma_{m}(a) \sigma_{m}(b)$. The first one is trivial from the definition of $\sigma_{m}$. Let $a=f_{a}(\theta)$ and $b=f_{b}(\theta)$ where $f_{a}(x), f_{b}(x) \in \mathbf{F}_{p}[x]$ has degree at most $r-1$. If $\operatorname{deg}\left(f_{a}(x) f_{b}(x)\right) \leq r-1$, it is easy to see that $\sigma_{m}(a b)=\sigma_{m}(a) \sigma_{m}(b)$. Now assume that $\operatorname{deg}\left(f_{a}(x) f_{b}(x)\right) \geq r$. Then $f_{a}(x) f_{b}(x)=h(x)+\left(x^{r}-a\right) p(x)$ where $h(x), p(x) \in \mathbf{F}_{p}[x]$ and $\operatorname{deg}(h(x))<r$. Then $\sigma_{m}(a b)=\sigma_{m}(h(\theta))=$ $h\left(\theta^{m}\right)=h\left(\theta^{m}\right)+\left(a^{m}-a\right) p\left(\theta^{m}\right)=h\left(\theta^{m}\right)+\left(\theta^{m r}-a\right) p\left(\theta^{m}\right)=f_{a}\left(\theta^{m}\right) f_{b}\left(\theta^{m}\right)=$ $\sigma_{m}(a) \sigma_{m}(b)$.

This shows that $\sigma_{m}$ is a homomorphism. To complete the proof, we need to show that it is also one-to-one. This is obvious since $\theta^{m}$ is a root of $x^{r}-a=0$.

Define $G_{m}=\left\{f(\theta) \in \mathbf{F}_{p}(\theta)^{*} \mid f\left(\theta^{m}\right)=f(\theta)^{m}\right\}$. It can be verified that $G_{m}$ is a group when $\sigma_{m}$ is in $\operatorname{Gal}\left(\mathbf{F}_{p}(\theta) / \mathbf{F}_{p}\right)$.

Lemma 4. Suppose $\sigma_{n} \in \operatorname{Gal}\left(\mathbf{F}_{p}(\theta) / \mathbf{F}_{p}\right)$. Then for any $i, j \geq 0, \sigma_{d^{i} p^{j}} \in$ $\operatorname{Gal}\left(\mathbf{F}_{p}(\theta) / \mathbf{F}_{p}\right)$ and $G_{n} \subseteq G_{d^{i} p^{j}}$.

Proof. Notice that the map $x \rightarrow x^{p^{l}}$ is a one-to-one map in $\mathbf{F}_{p}(\theta)$. The equation $a^{n}=a$ implies that $\left(a^{d}\right)^{p^{l}}=a$, hence $a^{d}=a$, and $a^{d^{i} p^{j}}=a$. We have $\sigma_{d^{i} p^{j}} \in$ $\operatorname{Gal}\left(\mathbf{F}_{p}(\theta) / \mathbf{F}_{p}\right)$.

Let $f(\theta) \in G_{n}$. Thus $f\left(\theta^{n}\right)=f(\theta)^{n}$, this implies $f\left(\theta^{p^{l} d}\right)=f(\theta)^{p^{l} d}=f\left(\theta^{p^{l}}\right)^{d}$. So $\theta^{p^{l}}$ is a solution of $f\left(x^{d}\right)=f(x)^{d}$. Since it is one of the conjugates of $\theta, \theta$ must be a solution as well. This proves that $f\left(\theta^{d}\right)=f(\theta)^{d}$. Similarly since $\theta^{d}$ is also one of the conjugates of $\theta$, as $\sigma_{d} \in \operatorname{Gal}\left(\mathbf{F}_{p}(\theta) / \mathbf{F}_{p}\right)$, we have $f\left(\theta^{d^{2}}\right)=f\left(\theta^{d}\right)^{d}=f(\theta)^{d^{2}}$. By reduction, $f\left(\theta^{d^{i}}\right)=f(\theta)^{d^{i}}$ for $k \geq 0$. Hence $f\left(\theta^{d^{i} p^{j}}\right)=f\left(\theta^{d^{i}}\right)=f(\theta)^{p^{j}} p^{j}$. This implies that $f(\theta) \in G_{d^{i} p^{j}}$. 
Lemma 5. If $\sigma_{m_{1}}, \sigma_{m_{2}} \in \operatorname{Gal}\left(\mathbf{F}_{p}(\theta) / \mathbf{F}_{p}\right)$ and $\sigma_{m_{1}}=\sigma_{m_{2}}$, then $\left|G_{m_{1}} \cap G_{m_{2}}\right|$ divides $m_{1}-m_{2}$.

This lemma is straight forward from the definition.

Lemma 6. Let $A=a^{r^{\alpha-1}}$. If $(1+\theta) \in G_{n}$, so is $1+A^{i} \theta$ for any $i=$ $1,2,3, \cdots, r-1$. And $\left|G_{n}\right| \geq 2^{r}$.

Proof. If $(1+\theta) \in G_{n}$, this means that $(1+\theta)^{n}=1+\theta^{n}$. It implies that $\left(1+\theta^{\prime}\right)^{n}=1+\theta^{\prime n}$ for any conjugate $\theta^{\prime}$ of $\theta$. Since $A$ is a primitive root of unity in $\mathbf{F}_{p}$, hence $A^{i} \theta$ are conjugates of $\theta$. We have $\left(1+A^{i} \theta\right)^{n}=1+\left(A^{i} \theta\right)^{n}=1+\left(A^{n}\right)^{i} \theta^{n}$ and we know that $A^{n}=A$. This proves that $1+A^{i} \theta \in G_{n}$. The group $G_{n}$ contains all the elements in the set

$$
\left\{\prod_{i=0}^{r-1}\left(1+A^{i} \theta\right)^{\epsilon_{i}} \mid \sum_{i=0}^{r-1} \epsilon_{i}<r\right\},
$$

by simple counting we have $\left|G_{n}\right| \geq 2^{r}$.

Finally we are ready to give the proof of the main theorem (Theorem 1) of this paper.

Proof. Since $\left|\operatorname{Gal}\left(\mathbf{F}_{p}(\theta) / \mathbf{F}_{p}\right)\right|=r$, hence there exist two different pairs $\left(i_{1}, j_{1}\right)$ and $\left(i_{2}, j_{2}\right)$ with $0 \leq i_{1}, j_{1}, i_{2}, j_{2} \leq\lfloor\sqrt{r}\rfloor$, such that $\sigma_{d^{i_{1}} p^{j_{1}}}=\sigma_{d^{i_{2}} p^{j_{2}}}$. According to Lemma 4, $G_{n} \subseteq G_{d^{i_{1}} p^{j_{1}}}, G_{n} \subseteq G_{d^{i_{2} p^{j_{2}}}}$, this implies that $G_{n} \subseteq G_{d^{i_{1}} p^{j_{1}}} \cap$ $G_{d^{i_{2}} p^{j_{2}}}$. Therefore $\left|G_{n}\right|$ divides $d^{i_{1}} p^{j_{1}}-d^{i_{2}} p^{j_{2}}$, but $d^{i_{1}} p^{j_{1}}-d^{i_{2}} p^{j_{2}}<n\lfloor\sqrt{r}\rfloor \leq$ $2^{\sqrt{r} \log n} \leq 2^{r}$. hence $d^{i_{1}} p^{j_{1}}-d^{i_{2}} p^{j_{2}}=0$, which in turn implies that $n$ is a power of $p$.

\section{Implementation and Conclusion}

In this paper, we propose a random primality proving algorithm which runs in heuristic time $\tilde{O}\left(\log ^{4} n\right)$. It generates a certificate of primality of length $O(\log n)$ which can be verified in deterministic time $\tilde{O}\left(\log ^{4} n\right)$.

When it comes to implement the algorithm, space is a bigger issues than time. Assume that $n$ has 1000 bit, which is in the range of practical interests. To compute $(1+x)^{n}\left(\bmod n, x^{r}-a\right)$, we will have an intermediate polynomial of size $2^{30}$ bit, or $128 \mathrm{M}$ bytes. As a comparison, ECPP is not very demanding on space. In order to make the algorithm available on a desktop PC, space efficient exponentiation of $1+x$ is highly desirable. This is the case for the original version of the AKS algorithm as well.

For the sake of theoretical clarity, we use just one round of ECPP reduction in the algorithm. To implement the algorithm, it may be better to follow the ECPP algorithm and launch the iteration of AKS as soon as an intermediate prime becomes good. Again assuming that the intermediate primes are distributed randomly, the expected number of rounds will be $\log \log n$. It is a better strategy since the intermediate primes get smaller. 
We can certainly incorporate small time-saving features suggested by various researchers on the original version of AKS. The details will be included in the full version of this paper.

Acknowledgments. We thank Professors Pedro Berrizbeitia and Carl Pomerance for very helpful discussions and comments.

\section{References}

1. L. M. Adleman and M.A. Huang. Primality Testing and Abelian Varieties Over Finite Fields. Lecture Notes in Mathematics. Springer-Verlag, 1992.

2. L. M. Adleman, C. Pomerance, and R. S. Rumely. On distinguishing prime numbers from composite numbers. Annals of Mathematics, 117:173-206, 1983.

3. M. Agrawal, N. Kayal, and N. Saxena. Primes is in P. http://www.cse.iitk.ac.in/news/primality.pdf, 2002.

4. A.O.L. Atkin. Lecture notes of a conference in Boulder (Colorado), 1986.

5. A.O.L. Atkin and F. Morain. Elliptic curves and primality proving. Mathematics of Computation, 61:29-67, 1993.

6. Eric Bach and Jeffrey Shallit. Algorithmic Number theory, volume I. The MIT Press, 1996.

7. Pedro Berrizbeitia. Sharpening "primes is in p" for a large family of numbers. http://lanl.arxiv.org/abs/math.NT/0211334, 2002.

8. S. Goldwasser and J. Kilian. Almost all primes can be quickly certified. In Proc. 18th ACM Symp. on Theory of Computing, pages 316-329, Berkeley, CA, 1986. ACM.

9. A. Lenstra and H. W. Lenstra Jr. Handbook of Theoretical Computer Science A, chapter Algorithms in Number Theory, pages 673-715. Elsevier and MIT Press, 1990 .

10. F. Morain. Primality proving using elliptic curves: An update. In Proceedings of ANTS III, volume 1423 of Lecture Notes in Computer Science, 1998.

11. G. Tenenbaum. Introduction to analytic and probabilistic number theory (English Translation). Cambridge University Press, 1995. 